\title{
Session 6: Infectious Diseases I
}

\author{
Thursday 15th April 2010. Moderators: Roberto Burioni and Mirek Gorny
}

\section{[10.30-11.00]}

'Potent human and Rhesus macaque neutralizing monoclonal antibodies that target the same quaternary epitope on intact HIV-1 virions'

Miroslaw K.Gorny, Xiao-Hong Wang, James Robinson and Susan Zolla-Pazner

New York University, New York, NY, USA

The nature and specificity of monoclonal antibodies (mAbs) depends on the method of selection of the Abproducing cells. Using a neutralization assay as screening tool, we were able to select Abs against HIV-1 that target structures found only on intact virus particles but not on soluble proteins.

A human mAb 2909 was generated from an HIV-1infected individual while Rhesus macaque mAbs were made from the cells of monkeys infected with SHIVSF162.P4 chimeric virus. The mAbs were produced using cellular techniques either based on fusion of EBVtransformed lymphocytes with heteromyeloma cells or from EBV-transformed cells only. A human anti-HIV mAb 2909 and 11 mAbs from three Rhesus macaques were selected on the basis of their neutralizing activity against HIV-1 $1_{S F 162}$ all of which recognized a similar complex epitope V2/V3 representing a quaternary structure on intact virions. The quaternary nature of the 2909 epitope was confirmed by binding of mAb 2909 to virus pseudotyped with SF162 envelope (Env) proteins expressed as trimers, but not to pseudovirus with Env containing a mutant form of gp41 which do not form trimers, but instead form gp120 monomers.

Both human and monkey mAbs exhibit remarkably potent activity against SF162 which can be explained by their ability to bind to the unliganded form of gp120 on the virus surface resulting in the blockade of virus from binding to the CD4 receptor and consequently to the receptor, CCR5. This conclusion was supported by experiments whereby mAb 2909 bound to SF162 wild type Env on transfected cells and inhibited sCD4 binding.
The immunonogenetic studies revealed that mAbs derived from each monkey are either unique or are somatic variants as classified on the basis of the CDR H3 sequence. Analysis of immunoglobulin ( $\mathrm{Ig}$ ) variable genes coding for the heavy and light chains unveiled that some of the Rhesus mAbs use the same lambda gene as human mAb 2909. This suggests that the quaternary epitope structure requires similar or identical shape of the antigen binding site determined by the Ig genes.

This new type of anti-HIV-1 Abs may be representative of other neutralizing Abs that target quaternary epitopes present only on trimers, an aspect of the humoral immune response against HIV-1 which still remains under-explored.

[11.00-11.30]

'Using the viral challenge models in both ferrets and humans to evaluate the use of antibodies against respiratory viruses'

Rob Lambkin-Williams, Anthony Gilbert, Alex Mann and John Oxford

Retroscreen Virology Ltd., London, UK

Abstract not provided.

\section{[11.30-12.00]}

\section{'Human monoclonal antibodies against influenza $\mathrm{A}$ viruses' \\ Roberto Burioni \\ Università Vita-Salute San Raffaele, Milano, Italy}

The role played by humoral response in controlling infection by influenza viruses has been repeatedly demonstrated in several clinical and experimental settings. However, the potential prophylactic or therapeutic role of monoclonal antibodies in this field have been argumented because of the high viral variability, and 
therefore because of the lack of molecules endowed with broadly neutralizing activity.

In this presentation, we describe two human monoclonal antibodies (HMabs), named PN-SIA49 and PNSIA-28 endowed with outstanding homosubtypic and heterosubtypic neutralizing activity, respectively. Both antibodies were obtained from of a 55-year-old patient, vaccinated with the seasonal vaccine and with a negative clinical history of infection with influenza virus in the past 10 years.

Both monoclonals were shown to be directed against hemagglutinin (HA), and to neutralize in different in vitro assays all tested $\mathrm{H} 1 \mathrm{~N} 1$ strains (A/Puerto Rico/8/34; A/Wilson-Smith/33; A/Malaya/302/54; A/ New Caledonia/20/99), including an isolate of the recent H1N1v/2009 strain (A/Milan/UHSR1/2009). For both HMabs IC50 against all tested isolates were in the subnanomolar range. Notably, cells transfected with the recombinant hemagglutinin (HA) gene of A/South Carolina/1/18 were recognized by both HMabs, thus suggesting that both PN-SIA28 and PN-SIA49 are directed against conformational epitopes extremely conserved on H1 HAs during the whole H1N1 pandemic period.

Even more interestingly, PN-SIA28 featured similar binding and neutralizing characteristics also against a broad panel of $\mathrm{H} 3 \mathrm{~N} 2$ subtype isolates (A/Hong Kong/8/68; A/Aichi/2/68; A/Port Chalmers/1/73; A/ Victoria/3/75), evidencing the presence of a similarly conserved epitope also in this highly phylogentically divergent subtype.

In conclusion, PN-SIA28 and PN-SIA49 are the evidence that broadly neutralizing antibodies are produced in the course of influenza infection. These molecules are good candidates for future in vivo prophylactic and therapeutic studies, and may be of great help for the identification of highly conserved epitopes to be used in new broad-range vaccinal strategies.

\section{[12.00-12.20]}

\section{'Human antibodies identify highly conserved func- tional epitopes on HIV-1 and influenza viruses' Matthew Moyle \\ Theraclone Sciences Inc., Seattle, Washington, USA}

Co-evolution with pathogens has led to adaptation by the human immune system of a remarkable array of protective strategies. To capture the humoral component of this evolved response, we have used a novel, highthroughput antibody discovery technology to identify directly from human B cells functional antibodies generated against HIV-1 and influenza. For HIV-1, monoclonal antibodies were isolated from an HIV-1-positive subject that neutralize more than $75 \%$ of a panel of 162 viruses across six viral clades. These HIV-1 antibodies bind conserved regions of variable loops of the gp120 subunit in the context of the trimeric Envelope protein. For influenza, we identified a family of related monoclonal antibodies from a cohort of healthy individuals that recognize a structure that is present on the surface of nearly all influenza A viruses. These influenza antibodies protect mice from a lethal challenge with a high-path avian H5N1 strain of influenza. Recombinant forms of these HIV-1 and influenza monoclonal antibodies are being evaluated as potential therapeutic candidates in preclinical models of disease. Additionally, the occurrence of anti-HIV-1 and -influenza antibodies that bind to highly conserved viral epitopes suggests that these respective structures are potentially immunogenic in humans. Such epitopes could therefore prove useful in the design of novel vaccines for HIV-1 and influenza.

\section{[12.20-12.40]}

'Characterization of a novel neutralizing monoclonal antibody that recognizes the fusion loop peptide of flavivirus envelope protein'

Yong-Qiang Deng, Guang-Hui Ji, Yan-Shen Kang, Tao Jiang, Man Yu, E-De Qin, Jian-Xin Dai, Ya-Jun Guo and Cheng-Feng Qin

Beijing Institute of Microbiology and Epidemiology, Beijing, China

Dengue, Japanese encephalitis, West Nile and yellow fever are major human pathogens that belong to the Flavivirus genus, and cause large epidemics and tens of thousands of deaths annually in many parts of the world. Given the lack of approved antiviral treatment, recombinant monoclonal antibodies (mAbs) have been verified as candidates for the prevention and treatment of flavivirus infections.

In this study, we firstly generated and characterized a panel of mAbs including 2A10G6 direct against dengue 2 virus prM-E protein. Indirect immunofluorescence assay found that mAb 2A10G6 were crossreactive with the viruses of the dengue group, and several other flaviviruses. Plaque reduction neutralization test determined that mAb 2A10G6 neutralized dengue $1-4$, West Nile and yellow fever viruses. In vivo protection experiments also showed that mAb 2A10G6 pro- 
tected sucking mice from lethal dengue 1-4 challenge with a dose-dependent manner. Thus, we established a flavivirus cross-reactive neutralizing mAb 2A10G6. Furthermore, phage-displayed random peptide library mapped the epitope of mAb 2A10G6 to a common antigenic site shared among different flaviviruses, including 98-RDXW-101 within the highly conserved Nterminal fusion loop peptide of the E protein domain II. Additionally, in vitro bind inhibition assays confirmed that mAb 2A10G6 blocked infection primarily at a step after viral attachment.

Together, these experiments define the characteristics of a novel flavivirus cross-reactive neutralizing mAb 2A10G6 and make it a suitable candidate for humanization into a therapeutic antibody to treat severe flavivirus infections in human.

[12.40-13.00]

'Plant production of antibodies for human fungal infection immunotherapy'

Antonio Cassone, C. Capodicasa, A. TorosantuccI, P. Chiani, C. bromuro, M. Catellani and E. Benvenuto

Istituto Superiore di Sanità, Rome, Italy

Human diseases caused by opportunistic fungal agents (in particular Candida albicans, Aspergillus spp and Cryptococcus neoformans) are dramatically in- creased in the recent years, causing high morbility and mortality mainly in immunocompromised and hospitalized hosts. A anti-ß-glucan monoclonal antibody (2G8 mAb) was previously generated in mice, which exerted a protective effect in vivo against experimental systemic aspergillosis and cryptococcosis as well as against disseminated and vaginal infections by Candida albicans. The VH and VL domains of this murine antibody have been engineered to obtain humanized anti-ß-glucan antibodies of different formats. Particularly, a recombinant antibody in the form of human chimeric full antibody and a ScFv-Fc version, have been transiently expressed in Nicotiana benthamiana plants and purified from leaves by protein A affinity chromatography with very high yields ( 40 and $50 \mathrm{mg} / \mathrm{kg}$ of plant tissue, respectively). Similarly to the protective murine $\mathrm{mAb}$ of origin, the human chimeric IgG and the $\mathrm{scFv}-\mathrm{Fc}$ plant expressed preferentially recognized $\beta$-glucan molecules in $\beta$ - $(1,3)$ configuration, bound $C$. albicans and $A$. fumigatus hyphae, were able to inhibit growth of $C$. albicans in vitro, in the absence of immune effectors, and prevented fungal adhesion to human epithelial cells. In conclusion, the engineered antibodies plant produced retain binding specificity and protection-relevant biological properties of the original $\mathrm{mAb}$ and, therefore, could represent potentially valuable tools for both diagnostics and immunoprophylaxis/therapy of human fungal diseases. 